\title{
Some insights into the migration of double imaginary roots under small deviation of two parameters
}

\author{
Dina Irofti ${ }^{\text {a }}$, Keqin $\mathrm{Gu}^{\mathrm{b}}$, Islam Boussaada ${ }^{\mathrm{a}}$, Silviu-Iulian Niculescu ${ }^{\mathrm{a}}$ \\ ${ }^{\text {a }}$ Laboratoire des Signaux et Systèmes (L2S) CNRS-CentraleSupélec-Université Paris-Sud, 3 rue Joliot-Curie 91192 \\ Gif-sur-Yvette cedex, France
}

${ }^{\mathrm{b}}$ Department of Mechanical and Industrial Engineering, Southern Illinois University Edwardsville, Edwardsville, Illinois 62026, USA

\begin{abstract}
This paper studies the migration of double imaginary roots of the systems' characteristic equation when two parameters are subjected to small deviations. The proposed approach covers a wide range of models. Under the least degeneracy assumptions, we found that the local stability crossing curve has a cusp at the point that corresponds to the double root, and it divides the neighborhood of this point into an S-sector and a G-sector. When the parameters move into the G-sector, one of the roots moves to the right half-plane, and the other moves to the left half-plane. When the parameters move into the S-sector, both roots move either to the left half-plane or the right half-plane depending on the sign of a quantity that depends on the characteristic function and its derivatives up to the third order.
\end{abstract}

Key words: Characteristic roots; Spectral analysis; Time delay; Stability analysis; Stability criteria.

\section{Introduction}

Control systems often depend on parameters and we may generally write their characteristic equation as

$$
q(s, p)=0
$$

where $s$ is the Laplace variable and $p \in \mathbb{R}^{n}$ is a vector of $n$ parameters. We can have parameters due to internal dynamics. For instance, modeling in physical, biological or social sciences sometimes requires taking into account the time delays inherent in the phenomena. Depending on the model complexity, but also on how much information is known, we may chose a model with continuous constant delays, or a model with distributed delays (see Cushing, 1977; MacDonald, 1989). For instance, in the case of a time-delay system with two constant delays, the characteristic equation can be written of the form

$$
q_{1}\left(s, \tau_{1}, \tau_{2}\right)=r_{0}(s)+r_{1}(s) e^{-\tau_{1} s}+r_{2}(s) e^{-\tau_{2} s},
$$

Email addresses: Dina. Irofti@l2s. centralesupelec.fr (Dina Irofti), kgu@siue.edu (Keqin Gu),

Islam.Boussaada@12s . centralesupelec.fr (Islam

Boussaada), Silviu.Niculescu@12s.centralesupelec.fr (Silviu-Iulian Niculescu). where $r_{k}(s), k=0,1,2$ are polynomials of $s$ with real coefficients, and the delays $\tau_{1}, \tau_{2}$ are the two parameters.

Also common is the case when $p$ contains controller parameters. Classical examples include PI, PD and PID controllers. For example, the continuous time PID controller is expressed in the Laplace domain as $q_{2}(s)=K_{P}\left(1+\frac{1}{T_{i} s}+T_{d} s\right)$, where $K_{P}$ is the proportional gain, $T_{i}$ and $T_{d}$ are the integral and derivative time constants. Furthermore, many process control problems also contain a time delay $\tau_{m}$ (see O'Dwyer, 2006; Morarescu, Mendez-Barrios, Niculescu \& Gu, 2011). These include proportional plus delay $q_{3}(s)$, integrator plus delay model $q_{4}(s)$, first order lag plus delay $q_{5}(s)$, first order lag plus integral plus delay $q_{6}(s)$ expressed below:

$$
\begin{aligned}
& q_{3}(s)=K_{m}\left(1+e^{-s \tau_{m}}\right) \quad q_{4}(s)=\frac{K_{m} e^{-s \tau_{m}}}{s} \\
& q_{5}(s)=\frac{K_{m} e^{-s \tau_{m}}}{1+s T_{m}} \quad q_{6}(s)=\frac{K_{m} e^{-s \tau_{m}}}{s\left(1+s T_{m}\right)}
\end{aligned}
$$

If in the expression of $q_{3}(s)$ there are two different gains for the two terms, then we obtain the proportional retarded controller: $q_{7}(s)=K_{p}+K_{r} e^{-s \tau_{m}}$. Furthermore, Villafuerte, Mondié \& Garrido (2013) showed 
that proportional retarded controller outperforms a PD controller on an experimental DC-servomotor setup. Obviously, any control among PID type results in a characteristic equation that depends on the control parameters.

Many studies have been conducted on the stability of systems that depend on parameters. For example, for systems with a single delay as the parameter, methods of identifying all the stable delay intervals are given in Lee \& Hsu (1969) and Walton \& Marshall (1987). For system with two parameters, a rich collection of stability charts (the parameter regions showing where the system is stable) for time-delay systems are presented in Stépán (1989). For systems with two delays as the parameters, a geometric approach is introduced in $\mathrm{Gu}$, Niculescu \& Chen (2005). This analysis is based on the continuity of the characteristic roots as functions of parameters (which needs to be carefully evaluated in the case of time delay systems of neutral type (see $\mathrm{Gu}, 2012$; Michiels \& Niculescu, 2007), and consists of identifying the parameters that correspond to imaginary characteristic roots and judging the direction of crossing of these roots as parameters change. Such an analysis is known as D-decomposition method (also known as Dsubdivision method). Such a method is first applied to time-delay systems in Neimark (1948). Earlier examples for other systems have been documented by the survey paper Gryazina, Polyak \& Tremba (2008). More recent studies using D-decomposition methods can be found in Ruan \& Wei (2003) and Gryazina \& Polyak (2006). Challenges due to non-differentiability arise when the imaginary roots are also multiple roots. Such problems have traditionally been solved using Puiseux series (Kato, 1980; Knopp, 1996), see, for example, Chen, Fu, Niculescu \& Guan (2010a) and Li, Niculescu, Çela, Wang \& Cai (2013) for systems with one parameter.

In this paper, we study systems with two parameters, and present a method to analyze the migration of roots in a neighborhood of the parameters corresponding to a double imaginary characteristic root. The method of analysis uses traditional complex analysis, and does not require Puiseux series. A preliminary version of this paper, which is restricted to the case of two point-wise delays as the parameters, was presented in Gu, Irofti, Boussaada \& Niculescu (2015). It should be pointed out that some phenomena discussed in this work, such as cusp in the parameter space, has also been presented in Levantovskii (1982). In this paper, we extend and generalize this method to a wide range of systems, as mentioned above, that can generally be written in the form of characteristic equation (1). Additionally, we illustrate how to apply the algebraic criterion by three examples.

\section{Problem statement and prerequisites}

Consider a system with the characteristic equation of the form $(1)$. For $p_{0}=\left(p_{10}, p_{20}\right)$, we assume that the function $q\left(s, p_{0}\right)$ has a double root on the imaginary axis, $s=s_{0}=i \omega_{0}$. In other words, we assume

$$
q\left(s_{0}, p_{0}\right)=\left.\frac{\partial q}{\partial s}\right|_{\substack{s=s_{0} \\ p=p_{0}}}=0
$$

We further assume that $s_{0}$ is not a third order root, i.e.

$$
\left.\frac{\partial^{2} q}{\partial s^{2}}\right|_{\substack{s=s_{0} \\ p=p_{0}}} \neq 0
$$

Suppose $q(s, p)$ is analytic with respect to $s$, and continuously differentiable with respect to $(s, p)$ up to the third order. We make the following additional non-degeneracy assumption:

$$
D=\operatorname{det}\left(\begin{array}{ll}
\operatorname{Re}\left(\frac{\partial q}{\partial p_{1}}\right) \operatorname{Re}\left(\frac{\partial q}{\partial p_{2}}\right) \\
\operatorname{Im}\left(\frac{\partial q}{\partial p_{1}}\right) \operatorname{Im}\left(\frac{\partial q}{\partial p_{2}}\right)
\end{array}\right) \begin{aligned}
& s=s_{0} \\
& \begin{array}{c}
s=p_{10} \\
p_{2}=p_{20}
\end{array}
\end{aligned} \neq 0
$$

where $\operatorname{Re}(\cdot)$ and $\operatorname{Im}(\cdot)$ denote the real and imaginary part of a complex number, respectively. Equations (3)-(5) will be the standing assumptions in the remaining part of this paper. Assumption (5) contains the first-order partial derivatives of $q$ with respect to the two parameters, $p_{1}$ and $p_{2}$.

Definition 1 For a system of the form (1) that satisfies (3), we say it is "the least degenerate" if assumptions (4)-(5) hold. We also say that inequalities (4) and (5) are the least degeneracy assumptions.

In view of the implicit function theorem, a consequence of inequality (5), which is one of the non-degeneracy assumptions, is that the characteristic equation (1) defines the pairs $\left(p_{1} p_{2}\right)$ in a small neighbourhood of the critical point $p_{0}=\left(\begin{array}{ll}p_{10} & p_{20}\end{array}\right)$ as a function of $s$ in a sufficiently small neighbourhood of $s_{0}$. Introduce the notation $\mathcal{N}_{\varepsilon}\left(x_{0}\right)=\left\{x|| x-x_{0} \mid<\varepsilon\right\}$ and $\mathcal{N}_{\varepsilon}^{\circ}\left(x_{0}\right)=$ $\left\{x|0<| x-x_{0} \mid<\varepsilon\right\}$ to denote the neighbourhood of a point $x_{0}$. Then, the above remarks can be more precisely stated in the following proposition.

Proposition 2 There exists an $\varepsilon>0$ and a sufficiently small $\delta>0$ such that for all $s \in \mathcal{N}_{\delta}\left(s_{0}\right)$, we may define $p_{1}(s)$ and $p_{2}(s)$ as the unique solution of (1) with $\left(p_{1}(s), p_{2}(s)\right) \in \mathcal{N}_{\varepsilon}\left(p_{10}, p_{20}\right)$. The functions so defined are differentiable up to the third order.

Note that, in general, for $s \in \mathcal{N}_{\delta}\left(s_{0}\right)$, characteristic equation (1) may have other solutions outside of $\mathcal{N}_{\varepsilon}\left(p_{10}, p_{20}\right)$. We recall the stability crossing curves defined in $\mathrm{Gu}$ et al. $(2005)$ as the set of all points $\left(p_{1}, p_{2}\right) \in \mathbb{R}_{+}^{2}$ such that 
$q(s)$ has at least one zero on the imaginary axis. Therefore, the set

$$
\begin{aligned}
& \mathcal{T}_{\left(\omega_{0}, p_{10}, p_{20}\right)}= \\
& \quad\left\{\left(p_{1}(i \omega), p_{2}(i \omega)\right) \in \mathcal{N}_{\varepsilon}\left(p_{10}, p_{20}\right) \mid i \omega \in \mathcal{N}_{\delta}\left(i \omega_{0}\right)\right\},
\end{aligned}
$$

which is a curve in the $p_{1}-p_{2}$ space that passes through the point $\left(p_{10}, p_{20}\right)$, is the restriction of stability crossing curves to a neighborhood of $\left(p_{10}, p_{20}\right)$. Thus, $\mathcal{T}_{\left(s_{0}, p_{10}, p_{20}\right)}$ will be known as the local stability crossing curve. Roughly speaking, it is a curve that divides the neighbourhood $\mathcal{N}_{\varepsilon}\left(p_{01}, p_{02}\right)$ of the parameter space into regions, such that the number of characteristic roots on the right half complex plane remains constant as the parameters vary within each such region. We also define the positive and negative local stability crossing curves, corresponding to $\omega>\omega_{0}$ and $\omega<\omega_{0}$, respectively. For instance, we use the notation $\mathcal{T}_{\left(\omega_{0}, p_{10}, p_{20}\right)}^{+}=\left\{\left(p_{1}(i \omega), p_{2}(i \omega)\right) \in \mathcal{N}_{\varepsilon}\left(p_{10}, p_{20}\right) \mid i \omega \in\right.$ $\left.\mathcal{N}_{\delta}\left(i \omega_{0}\right), \omega>\omega_{0}\right\}$ for the positive local stability crossing curve. We point out that the stability crossing curves are known as the D-decomposition curves in Gryazina \& Polyak (2006).

The purpose of this paper is to study how the two characteristic roots migrate as $\left(p_{1}, p_{2}\right)$ varies in a small neighborhood of $\left(p_{10}, p_{20}\right)$ under the least degeneracy assumptions.

\subsection{Cusp and local bijection}

We parametrize a neighbourhood of $s_{0}$ in the complex plane by using a radial variable $u$ and an angle $\theta: s=$ $s_{0}+u e^{i \theta}$. We also denote $\gamma=e^{i \theta}=\frac{\partial s}{\partial u}$. We can now fix the angular variable $\theta$, i.e., fix $\gamma$, and calculate the derivatives of $p_{1}$ and $p_{2}$ with respect to the radial variable $u$. This can be easily achieved by differentiating (1), yielding

$$
\frac{\partial q}{\partial p_{1}} \frac{\partial p_{1}}{\partial u}+\frac{\partial q}{\partial p_{2}} \frac{\partial p_{2}}{\partial u}+\frac{\partial q}{\partial s} \gamma=0
$$

If we set $u=0$ and use the second equation of (3) in (6), we obtain

$$
\left.\left(\begin{array}{l}
\operatorname{Re}\left(\frac{\partial q}{\partial p_{1}}\right) \operatorname{Re}\left(\frac{\partial q}{\partial p_{2}}\right) \\
\operatorname{Im}\left(\frac{\partial q}{\partial p_{1}}\right) \operatorname{Im}\left(\frac{\partial q}{\partial p_{2}}\right)
\end{array}\right) \begin{array}{c}
\substack{s=s_{0} \\
p_{1}=p_{10} \\
p_{2}=p_{20}} \\
\frac{\partial p_{1}}{\partial u} \\
\frac{\partial p_{2}}{\partial u}
\end{array}\right)_{u=0}=0,
$$

from which, given the non-degeneracy assumption (5), we conclude

$$
\left(\begin{array}{c}
\frac{\partial p_{1}}{\partial u} \\
\frac{\partial p_{2}}{\partial u}
\end{array}\right)_{u=0}=0 .
$$

Equation (7) has two important implications.
First, if we set $\gamma=i$, the equation (7) indicates that the local stability crossing curve $\mathcal{T}_{\left(\omega_{0}, p_{10}, p_{20}\right)}$ have a cusp at $\left(p_{10}, p_{20}\right)$ (see Guggenheimer, 1977). Indeed, as will be confirmed by considering the second-order derivative in the next subsection, $\mathcal{T}_{\left(\omega_{0}, p_{10}, p_{20}\right)}$ partitions a sufficiently small neighborhood of $\left(p_{10}, p_{20}\right)$ into a great sector (or G-sector) and a small sector ${ }^{1}$ (or S-sector) as shown in Figure 1. We will investigate how the double roots at $i \omega_{0}$ migrate as $\left(p_{1}, p_{2}\right)$ moves from $\left(p_{10}, p_{20}\right)$ to the $\mathrm{G}$-sector or the S-sector.



Fig. 1. G-sector and S-sector.

The second implication of the equation (7) may be stated as in the following corollary.

Corollary 3 Consider $s_{a} \in \mathcal{N}_{\delta}^{\circ}\left(s_{0}\right), \delta>0$ sufficiently small, and let $p_{1 a}=p_{1}\left(s_{a}\right), p_{2 a}=p_{2}\left(s_{a}\right)$ as defined in Proposition 2. Then equation (1) defines a bijection between $s$ in a small neighborhood of $s_{a}$ and $\left(p_{1}, p_{2}\right)$ in a small neighborhood of $\left(p_{1 a}, p_{2 a}\right)$.

Proof. We can show that $\left.\frac{\partial}{\partial s} q\left(s, p_{1 a}, p_{2 a}\right)\right|_{s=s_{a}} \neq 0$ (this is similar to Lemma 2 in Gu et al., 2015). Next, the implicit function theorem allows us to conclude that there exists a sufficiently small neighborhood of $\left(p_{1 a}, p_{2 a}\right)$ such that the equation (1) defines a unique function $s\left(p_{1}, p_{2}\right)$ with the function value restricted in a small neighborhood of $s_{a}$.

Obviously, the small neighborhood referred in Corollary 3 should not include $s_{0}$ and $\left(p_{10}, p_{20}\right)$ in view of the second condition of (3). Moreover, provided the continuity of solutions of the characteristic equation (1) with respect to the parameters $\left(p_{1}, p_{2}\right)$, Corollary 3 may be equivalently stated as follows.

Corollary 4 For all $\left(p_{1}, p_{2}\right) \in \mathcal{N}_{\varepsilon}^{\circ}\left(p_{10}, p_{20}\right)$ with $\varepsilon>0$ sufficiently small, the characteristic equation (1) has exactly two simple roots in a small neighborhood of $s_{0}$.

\footnotetext{
1 We have used the word "small" in a sense analogous to "small solution": a small sector is contained by a sector with straight sides with arbitrarily small angle when the neighborhood is sufficiently small.
} 


\subsection{Mapping in a neighborhood of a double root}

We can very clearly describe the mapping between $s$ and $\left(p_{1}, p_{2}\right)$ in the neighborhood of $s_{0}$ based on the second order derivative when $s-s_{0}$ is restricted to one quadrant. From this description, we can obtain the information on how the double root migrates as $\left(p_{1}, p_{2}\right)$ moves from $\left(p_{10}, p_{20}\right)$ to the G-sector or the S-sector in Figure 1 according to the sign of $D$, and whether the negative local stability crossing curve $\mathcal{T}_{\left(\omega_{0}, p_{10}, p_{20}\right)}^{-}$is on the clockwise side or on the counterclockwise side of $\mathcal{T}_{\left(\omega_{0}, p_{1}\right.}^{+}$ in the S-sector.

Taking a derivative of (6) with respect to the radial variable $u$, we obtain

$$
\begin{array}{r}
\frac{\partial^{2} q}{\partial p_{1}^{2}}\left(\frac{\partial p_{1}}{\partial u}\right)^{2}+2 \frac{\partial^{2} q}{\partial p_{1} \partial p_{2}} \frac{\partial p_{1}}{\partial u} \frac{\partial p_{2}}{\partial u}+2 \frac{\partial^{2} q}{\partial p_{1} \partial s} \frac{\partial p_{1}}{\partial u} \gamma+ \\
+\frac{\partial q}{\partial p_{1}} \frac{\partial^{2} p_{1}}{\partial u^{2}}+\frac{\partial^{2} q}{\partial p_{2}^{2}}\left(\frac{\partial p_{2}}{\partial u}\right)^{2}+2 \frac{\partial^{2} q}{\partial p_{2} \partial s} \frac{\partial p_{2}}{\partial u} \gamma+ \\
+\frac{\partial q}{\partial p_{2}} \frac{\partial^{2} p_{2}}{\partial u^{2}}+\frac{\partial^{2} q}{\partial s^{2}} \gamma^{2}=0
\end{array}
$$

Setting $u=0$ and applying (7) in (8), we arrive at

$$
\left[\frac{\partial q}{\partial p_{1}} \frac{\partial^{2} p_{1}}{\partial u^{2}}+\frac{\partial q}{\partial p_{2}} \frac{\partial^{2} p_{2}}{\partial u^{2}}+\frac{\partial^{2} q}{\partial s^{2}} \gamma^{2}\right]_{\substack{s=s_{0} \\ p_{1}=p_{10} \\ p_{2}=p_{20}}}=0
$$

The above can be solved for $\frac{\partial^{2} p_{1}}{\partial u^{2}}$ and $\frac{\partial^{2} p_{2}}{\partial u^{2}}$ to obtain,

$$
\begin{aligned}
& \left(\begin{array}{l}
\frac{\partial^{2} p_{1}}{\partial u^{2}} \\
\frac{\partial^{2} p_{2}}{\partial u^{2}}
\end{array}\right)_{\substack{s=s_{0} \\
p_{1}=p_{10} \\
p_{2}=p_{20}}}= \\
& -\left[\left(\begin{array}{l}
\operatorname{Re}\left(\frac{\partial q}{\partial p_{1}}\right) \operatorname{Re}\left(\frac{\partial q}{\partial p_{2}}\right) \\
\operatorname{Im}\left(\frac{\partial q}{\partial p_{1}}\right) \operatorname{Im}\left(\frac{\partial q}{\partial p_{2}}\right)
\end{array}\right)^{-1}\left(\begin{array}{l}
\operatorname{Re}\left(\frac{\partial^{2} q}{\partial s^{2}} \gamma^{2}\right) \\
\operatorname{Im}\left(\frac{\partial^{2} q}{\partial s^{2}} \gamma^{2}\right)
\end{array}\right)\right]_{\begin{array}{c}
s=s_{0} \\
p_{1}=p_{10} \\
p_{2}=p_{20}
\end{array}},
\end{aligned}
$$

which may also be written in a complex form

$$
\left(\begin{array}{c}
\frac{\partial^{2} p_{1}}{\partial u^{2}} \\
\frac{\partial^{2} p_{2}}{\partial u^{2}}
\end{array}\right)_{\substack{s=s_{0} \\
p_{1}=p_{10} \\
p_{2}=p_{20}}}=\frac{1}{D}\left(\begin{array}{c}
\operatorname{Im}\left(\frac{\partial q^{*}}{\partial p_{2}} \frac{\partial^{2} q}{\partial s^{2}} \gamma^{2}\right) \\
-\operatorname{Im}\left(\frac{\partial q^{*}}{\partial p_{1}} \frac{\partial^{2} q}{\partial s^{2}} \gamma^{2}\right)
\end{array}\right) \begin{gathered}
\substack{s=s_{0} \\
p_{1}=p_{10} \\
p_{2}=p_{20}} \\
.
\end{gathered}
$$

Given the equality (7), the tangent of the curve describing $\left(p_{1}, p_{2}\right)$ as a function of $u$ at $\left(p_{10}, p_{20}\right)$ is determined by the second order derivative given in equations (9) or (10). Before proceeding further, it is helpful to recall the following well known fact, which can be found in various elementary books that deal with geometry (see for example Gonzalez \& Stuart, 2008).
Lemma 5 Let $x^{(0)} \in \mathbb{R}^{2}$ and $M \in \mathbb{R}^{2 \times 2}$ be fixed. For any $x \in \mathbb{R}^{2}$, let $\theta$ be the angle to rotate $x^{(0)}$ to the $d i$ rection of $x$ in the counterclockwise direction. Let $\phi(\theta)$ be the angle to rotate $M x^{(0)}$ to the direction of $M x$ in the counterclockwise direction if $\operatorname{det}(M)>0$, and in the clockwise direction if $\operatorname{det}(M)<0$. Then the function $\phi(\theta)$ satisfies the following:

i) $\phi(\theta)$ is a continuous and increasing function of $\theta$

ii) $0<\phi(\theta)<\pi$ if and only if $0<\theta<\pi$.

We now make the following two observations about the second order derivative expression (9). First, set $\gamma=i$ and $\gamma=-i$, the expression determines the tangent of $\mathcal{T}_{\left(\omega_{0}, p_{10}, p_{20}\right)}$ as $\omega \rightarrow \omega_{0}$ from each side. As $\left(\frac{\partial^{2} p_{1}}{\partial u^{2}} \frac{\partial^{2} p_{2}}{\partial u^{2}}\right)^{T}$ given in (9) for $\gamma=i$ and $-i$ have the same value, $\mathcal{T}_{\left(\omega_{0}, p_{10}, p_{20}\right)}^{-}$and $\mathcal{T}_{\left(\omega_{0}, p_{10}, p_{20}\right)}^{+}\left(A^{\prime} C^{\prime}\right.$ and $C^{\prime} B^{\prime}$ in Figure $1)$ are tangent to each other at the point $\left(p_{10}, p_{20}\right)$, thus forming a cusp. Second, as $\gamma$ rotates through a $90^{\circ}$ angle in a counterclockwise direction, $\frac{\partial^{2} q}{\partial s^{2}} \gamma^{2}$ rotates through a $180^{\circ}$ angle in the same direction; and $\left(\frac{\partial^{2} p_{1}}{\partial u^{2}} \frac{\partial^{2} p_{2}}{\partial u^{2}}\right)^{T}$ given in (9) also rotates through a $180^{\circ}$ angle in a direction determined by the sign of $D$, which is the determinant of the matrix inverted: the rotation is counterclockwise if $D>0$, and it is clockwise if $D<0$ (according to Lemma 5).

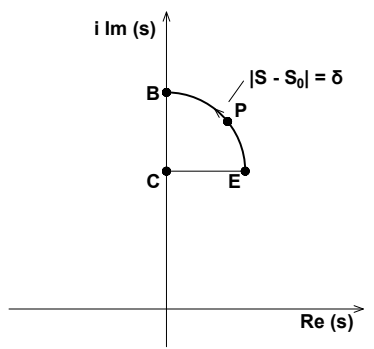

(a)



(b)
Fig. 2. The mapping $\left(p_{1}(s), p_{2}(s)\right)$ with $s-s_{0}$ in the first quadrant.

With the above observations, and the fact that

$$
\left(\begin{array}{l}
p_{1}(s) \\
p_{2}(s)
\end{array}\right)=\left(\begin{array}{l}
p_{10} \\
p_{20}
\end{array}\right)+\frac{u^{2}}{2}\left(\begin{array}{c}
\frac{\partial^{2} p_{1}}{\partial u^{2}} \\
\frac{\partial^{2} p_{2}}{\partial u^{2}}
\end{array}\right)_{\substack{s=s_{0} \\
p_{1}=p_{10} \\
p_{2}=p_{20}}}+o\left(u^{2}\right)
$$

we may describe the local mapping $\left(p_{1}(s), p_{2}(s)\right)$ in a very informative manner when $s-s_{0}$ is restricted to one quadrant. The situation for $s-s_{0}$ in the first quadrant

$$
Q_{1}=\left\{s=s_{0}+u e^{i \theta} \mid 0<u<\delta, 0 \leq \theta \leq \pi / 2\right\}
$$

with $D>0$ is illustrated in Figure 2: the line segment $C E$ (from $s_{0}$ to $s_{0}+\delta$ ) is mapped to the curve $C^{\prime} E^{\prime}$ in the 
$p_{1}-p_{2}$ space, the arc $\operatorname{EPB}\left(s=s_{0}+\delta e^{i \theta}, 0 \leq \theta \leq \pi / 2\right)$ is mapped to the curve $E^{\prime} P^{\prime} B^{\prime}$, and the line segment $B C$ (from $s_{0}+\delta i$ to $s_{0}$ ) is mapped to the curve $B^{\prime} C^{\prime}$. In view of the second order derivatives, $B^{\prime} C^{\prime}$ and $C^{\prime} E^{\prime}$ have the same tangent at $C^{\prime}$. Continuity and local bijectivity (Corollary 3 ) imply that the singly connected region bounded by the line segments $B C, C E$ and the arc $E P B$ is mapped by $\left(p_{1}(s), p_{2}(s)\right)$ bijectively to the singly connected region bounded by the curves $B^{\prime} C^{\prime}$, $C^{\prime} E^{\prime}$ and $E^{\prime} P^{\prime} B^{\prime}$. When $D<0$, the curve $E^{\prime} P^{\prime} B^{\prime}$ is roughly clockwise (instead of counterclockwise as in Figure 2) relative to the point $C^{\prime}$. The mapping with $s-s_{0}$ in the other three quadrants are similar.

\section{Main results}

The complete mapping $\left(p_{1}(s), p_{2}(s)\right)$ with $s-s_{0}$ in all four quadrants may be divided into four possible cases depending on the sign of $D$ and whether $\mathcal{T}_{\left(\omega_{0}\right.}^{-}$

is on the counterclockwise or on the clockwise $\left.\omega_{0}, p_{10}, p_{20}\right)$ $\mathcal{T}_{\left(\omega_{0}, p_{10}, p_{20}\right)}^{+}$in the S-sector. The migration of the double roots in all cases is summarized in the following theorem.

Theorem 6 (Migration of Double Roots) If $\left(p_{1}, p_{2}\right)$ is in the G-sector in a sufficiently small neighborhood of $\left(p_{10}, p_{20}\right)$, then one root of (1) in the neighborhood of $s_{0}$ is in the right half-plane, the other is in the left half-plane. When $\left(p_{1}, p_{2}\right)$ is in the S-sector, then the two roots are either both in the left half-plane or both in the right half-plane. More specifically,

Case $i$. If $D>0$ and $\mathcal{T}_{\left(\omega_{0}, p_{10}, p_{20}\right)}^{-}$is on the counterclockwise side of $\mathcal{T}_{\left(\omega_{0}, p_{10}, p_{20}\right)}^{+}$in the $S$-sector, then both roots are on the left half-plane.

Case ii. If $D>0$ and $\mathcal{T}_{\left(\omega_{0}, p_{10}, p_{20}\right)}^{-}$is on the clockwise side of $\mathcal{T}_{\left(\omega_{0}, p_{10}, p_{20}\right)}^{+}$in the $S$-sector, then both roots are on the right half-plane.

Case iii. If $D<0$ and $\mathcal{T}_{\left(\omega_{0}, p_{10}, p_{20}\right)}^{-}$is on the counterclockwise side of $\mathcal{T}_{\left(\omega_{0}, p_{10}, p_{20}\right)}^{+}$in the $S$-sector, then both roots are on the right half-plane.

Case iv. If $D<0$ and $\mathcal{T}_{\left(\omega_{0}, p_{10}, p_{20}\right)}^{-}$is on the clockwise side of $\mathcal{T}_{\left(\omega_{0}, p_{10}, p_{20}\right)}^{+}$in the $S$-sector, then both roots are on the left half-plane.

Proof. Consider Case i. The situation is illustrated in Figure 3. Let the region bounded by the arc EPB and line segments $B C$ and $C E$ be denoted as $I$, and the region bounded by the curves $E^{\prime} P^{\prime} B^{\prime}, B^{\prime} C^{\prime}$ and $C^{\prime} E^{\prime}$ be denoted as $I^{\prime}$. Similarly, region $I I$ is bounded by $B Q F$, $F C, C B$, and region $I I^{\prime}$ is bounded by $B^{\prime} Q^{\prime} F^{\prime}, F^{\prime} C^{\prime}$, $C^{\prime} B^{\prime}$; region $I I I$ is bounded by $F R A, A C, C F$, and $I I I^{\prime}$ is bounded by $F^{\prime} R^{\prime} A^{\prime}, A^{\prime} C^{\prime}, C^{\prime} F^{\prime}$; region $I V$ is

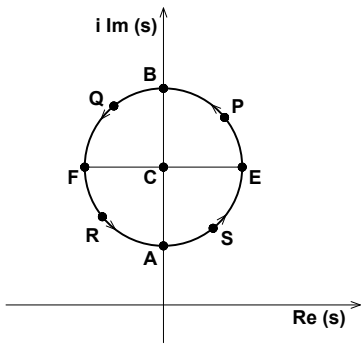

(a)



(b)
Fig. 3. The mapping $\left(p_{1}(s), p_{2}(s)\right)$ in a neighborhood of $s_{0}$. Case i: $D>0$, and $\mathcal{T}_{\left(\omega_{0}, p_{10}, p_{20}\right)}^{-}$is on the counterclockwise side of $\mathcal{T}_{\left(\omega_{0}, p_{10}, p_{20}\right)}^{+}$in the S-sector.

bounded by $A S E, E C, C A$, and region $I V^{\prime}$ is bounded by $A^{\prime} S^{\prime} E^{\prime}, E^{\prime} C^{\prime}, C^{\prime} A^{\prime}$. As discussed before the theorem, $\left(p_{1}(s), p_{2}(s)\right)$ is a bijection from $I$ to $I^{\prime}$ when $s$ is restricted to $I$. Similarly, $\left(p_{1}(s), p_{2}(s)\right)$ is a bijection from $I I$ to $I I^{\prime}$ when restricted to $I I$, or from $I I I$ to $I I I^{\prime}$ when restricted to $I I I$, or from $I V$ to $I V^{\prime}$ when restricted to $I V$. As the S-sector (in a sufficiently small neighborhood) is contained in $I I^{\prime} \cap I I I^{\prime}$, we may conclude that for any $\left(p_{1}, p_{2}\right)$ in the S-sector, one of the two characteristic roots in the neighborhood of $s_{0}$ must be in region $I I$, the other must be in region $I I I$, and obviously both in the left half-plane. Similarly, the G-sector (in a sufficiently small neighborhood) is contained in $\left(I^{\prime} \cup I V^{\prime}\right) \cap\left(I I^{\prime} \cup I I I^{\prime}\right)$. Therefore, for any $\left(p_{1}, p_{2}\right)$ in the G-sector, one of the two characteristic roots in the neighborhood of $s_{0}$ must be in $I \cup I V$ (in the right half-plane), and the other must be in $I I \cup I I I$ (in the left half-plane). Other three cases can be proved in a similar manner.

Theorem 6 indicates that the migration pattern of the two roots in the G-sector is always the same under the least degeneracy assumptions, which is the only case discussed in this article. However, judging the migration pattern of the two roots in the S-sector requires knowing the sign of $D$ and on which side of $\mathcal{T}_{\left(\omega_{0}, \tau_{10}, \tau_{20}\right)}^{+}$the curve $\mathcal{T}_{\left(\omega_{0}, \tau_{10}, \tau_{20}\right)}^{-}$is in the S-sector. Fortunately, by considering the third order derivatives, an explicit algebraic condition is possible. Such an explicit condition can be very convenient for designing control systems with two parameters.

Corollary 7 (Algebraic S-sector Criterion) Consider a control system of the form (1) with two parameters (i.e. $p$ vector is of dimension two), with a double characteristic root on the imaginary axis at $s=s_{0}$, for $p_{0}=\left(p_{10}, p_{20}\right)$. Suppose that the system is the least degenerate. When a small perturbation affects the parameter pair, if $\left(p_{1}, p_{2}\right)$ is in the $S$-sector in a sufficiently small neighborhood of $\left(p_{10}, p_{20}\right)$, then the two characteristic roots in the neighborhood of $s_{0}$ are both in the left 
half-plane if $\kappa<0$, where

$\kappa=\operatorname{Re}\left[\frac{\partial^{2} q}{\partial s^{2}}\left(-\frac{\partial^{3} q}{\partial s^{3}}+3 \frac{\partial^{2} q}{\partial p_{1} \partial s} \frac{\partial^{2} p_{1}}{\partial u^{2}}+3 \frac{\partial^{2} q}{\partial p_{2} \partial s} \frac{\partial^{2} p_{2}}{\partial u^{2}}\right)\right]$

evaluated at $s=s_{0},\left(p_{1}, p_{2}\right)=\left(p_{10}, p_{20}\right), \gamma=i$, and $\frac{\partial^{2} p_{i}}{\partial u^{2}}$ can be evaluated by (10) or (9) with $\gamma=i$. If $\kappa>0$ instead, then both roots are in the right half-plane.

The proof of the above corollary is based on Theorem 6 , and it is very similar to that of Corollary 8 in Gu et al. (2015), thus it is omitted here due to space limitation.

If $\kappa=0$, higher order derivatives may be used to evaluate conditions in Theorem 6. Note that the roots of the characteristic equation discussed in Theorem 6 and Corollary 7 are restricted to a sufficiently small neighborhood of $s_{0}=j \omega_{0}$. Because characteristic roots are distributed symmetrically with respect to the real axis, there is also a double root at $s_{0}^{*}=-j \omega_{0}$ when $p_{1}=p_{10}$ and $p_{2}=p_{20}$. When $\left(p_{1}, p_{2}\right)$ deviates from $\left(p_{10}, p_{20}\right)$, the migration of the two roots in the neighborhood of $s_{0}^{*}$ follows the same pattern as those in the neighborhood of $s_{0}$. There may also be roots on the imaginary axis outside the neighborhoods of $s_{0}$ and $s_{0}^{*}$. The migration of these imaginary roots needs to be analyzed separately. Finally, the roots on the right half-plane remain on the right half-plane as long as $\left(p_{1}, p_{2}\right)$ stay within a sufficiently small neighborhood of $\left(p_{10}, p_{20}\right)$. Similarly, the roots on the left half-plane remain on the left half-plane when the deviation of $\left(p_{1}, p_{2}\right)$ is sufficiently small.

\section{Illustrative examples}

In this section, we will present two examples to illustrate the application of the theory, as well as one degenerate cases illustrating that the least degeneracy assumptions are important. Consider the characteristic equation

$$
s^{5}+p_{1} s^{4}+p_{2} s^{3}+p_{1}^{2} s^{2}+s+2=0,
$$

where $p_{1}$ and $p_{2}$ are real parameters. For $\left(p_{1}, p_{2}\right)=(2,2)$, systems (11) has double imaginary roots at $s= \pm s_{0}=$ $\pm i \omega_{0}$, where $\omega_{0}=1$. In addition, it has a root at -2 , which is in the left half-plane. The local stability crossing curve $\mathcal{T}_{(1,2,2)}$ is plotted in Figure 4 , where $C^{\prime} A^{\prime}$ is $\mathcal{T}_{(1,2,2)}^{-}$, and $C^{\prime} B^{\prime}$ is $\mathcal{T}_{(1,2,2)}^{+}$. We can compute $\kappa=-128<0$. According to Corollary 7 , this means that both roots at $i$ moves to the left half-plane as $\left(p_{1}, p_{2}\right)$ moves into S-sector. Furthermore, we may compute $D=3>0$. Therefore, the system (11) belongs to Case i of Theorem 6 , i.e. $\mathcal{T}_{\left(\omega_{0}, p_{10}, p_{20}\right)}^{-}$is on the counterclockwise side of $\mathcal{T}_{\left(\omega_{0}, p_{10}, p_{20}\right)}^{+}$in the S-sector, which is consistent with Figure 4 . Also according to Theorem 6 , as $\left(p_{1}, p_{2}\right)$ moves from $(2,2)$ to the G-sector, one of the two imaginary roots at $i$ moves to the right half-plane, and the other



Fig. 4. $p_{1}-p_{2}$ parameter space for Equation (11).

one moves to the left half-plane. The movement of the double roots at $-i$ is symmetric to those at $i$. To summarize, for $\left(p_{1}, p_{2}\right)=(2,2)$, the system has four roots on the imaginary axis and one root on the left half-plane. When $\left(p_{1}, p_{2}\right)$ moves into the S-sector, all five roots are on the left half-plane. When $\left(p_{1}, p_{2}\right)$ moves into the G-sector, there are two roots on the right half-plane, and the remaining three roots are on the left half-plane.

Distributed delays also appear in many practical systems. An early example is given by Cushing (1977) to model population dynamics as follows

$$
\dot{x}(t)=a x(t)+b \int_{-\sigma}^{0} w(\theta) x(t+\theta) d \theta,
$$

where $w(\theta)$ is the kernel function. In chapter 2 of Insperger \& Stepan (2011), the special case of $w(\theta) \equiv 1$ was studied. In this case, the characteristic function becomes

$$
q(s)=s-a-b \frac{1-e^{-s \sigma}}{s}, \quad s \neq 0 .
$$

Next example considers the case with two such distributed delays. Consider the "Cushing-like" system with the following characteristic quasi-polynomial:

$$
q\left(s, \tau_{1}, \tau_{2}\right)=s-a-b \frac{1-e^{-s \tau_{1}}}{s}-c \frac{1-e^{-s \tau_{2}}}{s},
$$

where $a=-0.214104, b=-0.996801$, and $c=0.5$. System (13) has double imaginary roots at $s_{0}= \pm i \omega_{0}$ with $\omega_{0}=1$ for $\tau_{1}=\tau_{10} \approx 3.8403026849$ and $\tau_{2}=\tau_{20} \approx 10.44866732901$. We compute $D$ and $\kappa$ to obtain $D \approx 0.159228>0$, and $\kappa \approx-105541<0$. Judging from the sign of $D$ and $\kappa$, we can see that this example belongs to Case $\mathrm{i}$ in Theorem 6, i.e. $D>0$ and $\mathcal{T}_{\left(\omega_{0}, \tau_{10}, \tau_{20}\right)}^{-}$is on the counterclockwise side of $\mathcal{T}_{\left(\omega_{0}, \tau_{10}, \tau_{20}\right)}^{+}$in the S-sector. The stability crossing curve 


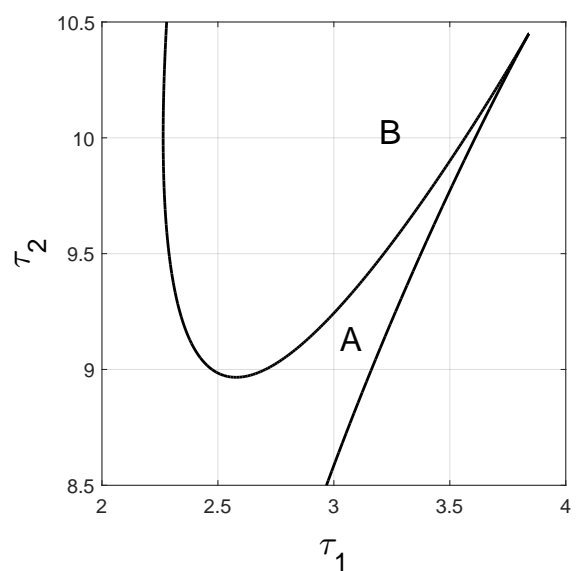

Fig. 5. $\tau_{1}-\tau_{2}$ parameter space for Equation (13). Characteristic equation (13) has no roots on the right half-plane when $\left(\tau, \tau_{2}\right)$ is in region $\mathrm{A}$ and two roots with positive real part when $\left(\tau_{1}, \tau_{2}\right)$ is in region $\mathrm{B}$.

$\mathcal{T}$ is depicted in Figure 5. We remark that $\mathcal{T}$ divides this area into two regions: region A containing S-sector and the origin, and region B containing the G-sector. For $\tau_{1}=\tau_{2}=0$, the characteristic quasi-polynomial reduces to a polynomial that has only one root equal to $a$. As $a$ is negative, we conclude that for $\left(\tau_{1}, \tau_{2}\right)$ in region $\mathrm{A}$, the quasi-polynomial (13) has no root with positive real part, and the system is stable. Next, according to Corollary 7 or Theorem 6 , both imaginary roots at $i$ move to the left half-plane as $\left(\tau_{1}, \tau_{2}\right)$ moves from the cusp to the S-sector (region A). Furthermore, according to Theorem 6 , as $\left(\tau_{1}, \tau_{2}\right)$ moves to G-sector (region $\mathrm{B}$ ), one of the imaginary roots moves to the right half-plane, and the other one to the left halfplane. In other words, as $\left(\tau_{1}, \tau_{2}\right)$ moves from region $\mathrm{A}$ to region $\mathrm{B}$ through $\left(\tau_{10}, \tau_{20}\right)$, one of the two imaginary roots moves from the left half-plane to the right half-plane passing through the point $i$ of the imaginary axis, and the other root moves in the left half-plane to touch the imaginary axis at $i$ and then returns to the left half-plane. Due to symmetry, another left half-plane root moves to the right half-plane through the point $-i$. Thus, in region $\mathrm{B}$, there are two more roots with positive real part, as compared to the region A. Thus, we conclude that there are two roots on the right half-plane when $\left(\tau_{1}, \tau_{2}\right)$ is in region $\mathrm{B}$.

In the sequel, we presented a degenerate case, where the local stability crossing curve may not have a cusp when one of the least degeneracy assumptions, $D \neq 0$, is violated. Consider the characteristic equation

$$
s^{5}+s^{4}+p_{2} s^{3}+\left(p_{1}+1\right) s^{2}+s+p_{1}=0,
$$

where $p_{1}$ and $p_{2}$ are real parameters. For $p_{1}=1$ and $p_{2}=2,(14)$ has a double root at $s_{0}= \pm i \omega_{0}$ with $\omega_{0}=1$. We can compute $D=0$, and therefore assumption (5) is



Fig. 6. $p_{1}-p_{2}$ parameter space for Equation (14). Point $\left(p_{10}, p_{20}\right)=(1,2)$ corresponds to double root at $\omega_{0}=1$. $D=0$. The stability crossing curve $\mathcal{T}$ does not have a cusp.

violated. The local stability crossing curve is plotted in Figure 6. It can be seen that there is no cusp at $(1,2)$, and S-sector and G-sector are not well defined.

\section{Concluding remarks}

The migration of double imaginary roots of characteristic equations that depend on two parameters is studied under the least degeneracy assumptions. It is shown that in the parameter space, the local stability crossing curve has a cusp and divides the neighbourhood of the critical point into two regions: an S-sector and a G-sector. As the parameter pair moves to the G-sector, one root moves to the left half-plane and the other moves to the right half-plane. If the parameter pair moves to the $\mathrm{S}$ sector, a simple algebraic criterion may be used to judge whether both roots move to the right half-plane or the left half-plane.

\section{References}

Barmish, B.R. (1993). New Tools for Robusteness of Linear Systems. Macmillan Coll Div.

Chen, J., Fu, P., Niculescu, S. I., \& Guan, Z. (2010a). An eigenvalue perturbation approach to stability analysis, part 1: eigenvalue series of matrix operators. SIAM Journal of Control Optimization, 48(8), 5564-5582.

Cushing, J.M. (1977). Time delays in single species growth models. Journal of Mathematical Biology, 4(3), $257-264$.

Gonzalez, O., \& Stuart, A. M. (2008). A First Course in Continuum Mechanics. Cambridge University Press.

Gryazina, E. N., \& Polyak, B. T. (2006). Stability regions in the parameter space: D-decomposition revisited. Automatica, 42(1), 13-26.

Gryazina, E. N., Polyak, B. T., \& Tremba, A. A. (2008). D-decomposition technique state-of-the-art. Automation and Remote Control, vol. 69, no. 12, pp. 19912026.

$\mathrm{Gu}, \mathrm{K}$. (2012). A review of some subtleties of practical relevance for time-delay systems of neutral type. ISRN 
Applied Mathematics, Vol. 2012, Article ID 725783, 46 pages. doi:10.5402/2012/725783.

Gu, K., Irofti, D., Boussaada, I., \& Niculescu, S.-I. (2015). Migration of double imaginary characteristic roots under small deviation of two delay parameters. CDC Conference on Decision and Control, pp. 64106415. DOI 10.1109/CDC.2015.7403229.

Gu, K., Niculescu, S.-I., \& Chen, J. (2005). On stability of crossing curves for general systems with two delays. J. Math. Anal. Appl., vol. 311, pp. 231-253.

Guggenheimer, H. W. (1977). Differential Geometry. New York: Dover.

Insperger, T., \& Stepan, G. (2011). Semi-discretization method for delayed systems. Springer.

Kato, T. (1980). Perturbation Theory for Linear Operators. 2nd Ed, Springer-Verlag, Berlin.

Knopp, K. (1996). Theory of Functions. Parts I and II, Translated to English by F. Bagemihl, Dover, Mineola.

Kolmanovskii, V., \& Myshkis, A. (1999). Introduction to the Theory and Applications of Functional Differential Equations. Kluwer, Dordrecht, the Netherlands.

Lee, M. S., \& Hsu, C. S. (1969). On the $\tau$-decomposition method of stability analysis for retarded dynamical systems. SIAM J. Control, 7:249-259.

Levantovskii, L. V. (1982). Singularities of the boundary of the stability domain. Functional analysis and its applications, 16(1), 34-37.

Li, X.-G., Niculescu, S.-I., Çela, A., Wang, H.-H., \& Cai, T.-Y. (2013). On computing Puiseux series for multiple imaginary characteristic roots of LTI systems with commensurate delays. IEEE Trans. Autom. Control, 58, 1338-1343.

MacDonald, N. (1989). Biological delay systems: linear stability theory. Cambridge University Press, Cambridge.

Mailybaev, A. A. (2000). On stability of polynomials depending on parameters. Journal of Computer and Systems Sciences International, vol. 39, no. 2.

Michiels, W., \& Niculescu, S.-I. (2014). Stability and stabilization of time-delay systems. An eigenvalue based approach. SIAM: Philadelphia, USA, Advances in design and control, vol. 27.

Morarescu, I.-C., Mendez-Barrios, C., Niculescu, S.-I., \& Gu, K. (2011). Stability crossing boundaries and fragility characterization of PID controllers for SISO systems with I/O Delays. American Control Conference, $A C C$ 2011, San Francisco, United States. pp.4988-4993.

Neimark, Yu. I. (1948). The structure of the Ddecomposition of the space of quasipolynomials and the diagrams of Vysnegradskii and Nyquist (in Russian). Dokl. Akad. Nauk SSSR, vol. 60, pp. 1503-1506.

O'Dwyer, A. (2006). PI and PID controller tuning rules: an overview and personal perspective. Proceedings of the IET Irish Signals and Systems Conference, pp. $161-166$.

Ruan, S., \& Wei, J. (2003). On the zeros of transcendental functions with applications to stability of delay differential equations with two delays. Dynamics of Continuous Discrete and Impulsive Systems Series A, 10, 863-874.

Stépán, G. (1989). Retarded Dynamical Systems: Stability and Characteristic function. Wiley, New York.

Villafuerte, R., Mondié, S., \& Garrido, R. (2013). Tuning of Proportional Retarded Controllers: Theory and Experiments. Transactions on Control SystemsTechnology (TCST-IEEE), Vol. 21, N. 3, pp 983-990.

Walton, K. \& Marshall, J. E. (1987). Direct method for TDS stability analysis. Proc. IEE, 134(2):101-107. 